in this field remains the failure by local authorities to provide hostels and other accommodation and services that would allow patients to be discharged from hospital to the community. This would also relieve the problems inside the hospitals caused by overcrowding. At the last count there were 294 patients in South Ockenden whom the staff thought fit for discharge if only care could have been provided for them in the community. Little wonder, then, that in the words of the report "there has grown up amongst many who work at the hospital an unhelpful but inevitable cynicism as they have observed that the exhortation to local authorities to build hostels has been unaccompanied by sufficient money to do more than scratch the surface of the need."

During the Parliamentary debate on the report, ${ }^{6} \mathrm{Mrs}$. Castle said that since 1969 the numbers of patients in these hospitals has gone down by 7,000 but the number of local authority places has risen in the same period by only 4,000 ; and that "within the overall central and local government expenditure limits" for the health and personal social services she would press for priority to be given to services for the mentally handicapped. This is really no answer at all. Already the health services have been placed in great difficulties by the cuts in their budgets made by the last administration at the time of the oil crisis $;^{7}$ the full effects of these cuts are only now becoming apparent. ${ }^{8}$ If the only way money can be found for the mentally handicapped is to take it from services for the acutely ill then these services will simply collapse more quickly.

For the acute services are on the point of collapse: the revolt by the nurses is evidence of that. Politicians have glibly told the public that the numbers of nurses have been rising and they have-the total hospital nursing staff in Britain rose from 280,000 in 1966 to 350,000 in 1972 . During that time however, hospital work has become much more complicated and has needed many more staff. Intensive care units, dialysis units, premature baby units, and similar specialist departments require a high nurse-patient ratio-and they also require trained, registered nurses, not simply pairs of hands. While the total numbers of nurses have been rising the proportion of S.R.N.s has been falling: as nursing duties have become more specialist the personnel have become less fully trained. ${ }^{9}$ Furthermore, nurses are justifiably concerned about the burden of routine work being placed on students-it is common experience at night to find a ward in the charge of a student nurse. The fundamental problem, however, is pay. At present an experienced ward sister or charge nurse earns no more than $£ 1$ an hour-and less than this when first appointed. The staff nurses' scale ranges from $64 p$ to $74 p$ an hour (all rates based on a 40 hour week). After deductions for tax, insurance, and pension many young nurses take home less than $£ 20$ a week. How can they hope to find accommodation and live at a reasonable standard?

No-one pretends that inadequate pay is the only problem for nurses. The Salmon career structure penalizes the nurses who wishes to make a career as a ward-sister; and there is still too little effort made to make the best use of trained nurses who are married and wish to work part-time. In the same way, doctors' grievances, especially in the hospital service, are not solely concerned with pay. But for both groups the effect on morale of the seeming indifference of the authorities to their pay claims and of the public to declining standards, has a corrosive effect on any discussion of other N.H.S. problems.

N.H.S. reorganization has hardly yet got under way, and if it is to work at all it will need a spirit of co-operation and enthusiasm from all concerned. This will not be forthcoming if the Government's response to the current upsurge of dissatisfaction is repetition of tired platitudes about priorities and resources. The situation is simple: if the N.H.S. is to retain adequate staff of high quality it must pay them properly, and that means either giving health a bigger share of the national cake or abandoning the concept of a free comprehensive service and making some cuts in the provision of medical care. Switching resources around "within the overall limits of government expenditure on health" will get the country nowhere.

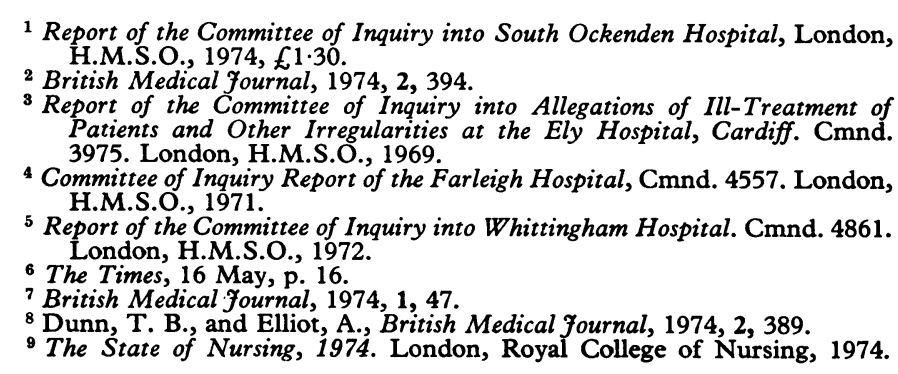

\section{Simulated Patients}

Recently Dr. Howard Barrows, a neurologist from the new medical school at McMaster University, visited London to conduct a two day workshop on simulated patients arranged by the Department of Audiovisual Communications on behalf of the B.M.A. Board of Science. He brought with him a young nurse on the neurological service at McMaster who has been responsible for training the volunteers being used as simulated patients in the educational programme there. Her simulations of illness were highly convincing: her repetoire ranged from simple depression to unconsciousness with decerebrate rigidity. Dr. Barrows's own interests lie in neurological, locomotor, and psychiatric diseases and it was from these fields that most of the conditions derived. Neurological simulations are particularly impressive as physical signs can be reproduced with great accuracy. The features of diseases in other systems can also be simulated, however, particularly if the most important pointers are obtainable from the history, and it was surprising how well some physical signs in the chest or abdomen could be mimicked.

Dr. Barrows believes that simulated patients can be used for a large number of different educational purposes. ${ }^{1} \mathrm{He}$ stresses that simulation should not be thought of as a substitute for real patients, but his enthusiasm may have led him to over-play his use of this technique in teaching about common clinical problems. Few experienced medical teachers have serious difficulties relating to the reactions of real patients either to being interviewed by students or to being discussed at the bedside. Indeed when used skilfully bedside teaching can help to reassure a patient about the quality of the care he is receiving and give him an insight into the nature and consequences of his illness.

The use of simulated patients for clinical teaching or for the evaluation of students and postgraduates may not appeal immediately to British clinical teachers, but the idea should not be condemned out of hand. There are a number of clinical situations which need particularly delicate handling, and an awkward or ill-timed approach during interview might have a damaging effect on the patient or cause considerable embar- 
rassment. This can be true in the care of the dying or if patients have severe or unusual disturbances of mood or behaviour; problems may also arise when asking questions of a highly personal nature or in dealing with patients who are hostile or sexually threatening. Allowing students to examine patients who are very ill or those awaiting litigation is also potentially dangerous. Clearly students must learn to handle such situations, and it seems common sense to allow them to practise their parts in conditions in which no damage can be done. For this purpose simulated patients would be excellent substitutes.

The major problem about the use of simulated patients must be the logistics. Large numbers of individuals capable of playing one or more parts would be needed if the technique is to be available to all of the students entering medical school each year. The training process itself seems to be no problem; medical members of the workshop had no difficulty in teaching simulation to several lay people who were also in attendance. There would, however, need to be an organization within the medical school to control the training programme, and the subjects themselves would probably have to be paid, though drama schools or other interested groups might be prepared to help on a voluntary basis. The cost might be formidable. Despite these obstacles some of the 13 medical schools represented at the workshop may well decide to assess the use of simulated patients in their own environment. Used sensibly, they could provide a valuable addition to the hardy professional patients who return to hospital at the start of each academic year.

1 Barrows, H. S., Simulated Patients (Programmed Patients)-The Development and Use of a New Technique in Medical Education. Thomas, Illinois, 1971 .

\section{Tetracyclines after 25 Years}

The discovery of Aureomycin (chlortetracycline) in 1948 was the result of a systematic screening programme for antibiotic activity in a large number of Actinomycetales, and the new species from which it was extracted was named Streptomyces aureofaciens because its colonies were golden-yellow. Though chloramphenicol had just been discovered the only antibiotics in general use at that time were penicillin and streptomycin. Aureomycin and chloramphenicol had two great advantages: they could be given by mouth and they had a broad spectrum of antimicrobial activity. The wide use of chloramphenicol became limited by the rare but extremely serious risk of bone marrow depression. Unwanted effects of chlortetracycline were less serious and, together with its successors oxytetracycline and tetracycline, the early tetracyclines came into wide general use.

Tetracyclines are active against the large majority of Grampositive and Gram-negative cocci and bacilli, both aerobic and anaerobic. Proteus and Pseudomonas species form the main exceptions. Tetracyclines are also active against a remarkably wide range of other organisms, including treponemata, mycoplasma, chlamydia, and rickettsias. This genuinely broad spectrum of activity made the tetracyclines extremely popular, since they could be used in good conscience for very many patients in whom bacterial infection was suspected but in whom, as so often, the precise causal diagnosis was unknown.

The first three tetracyclines held sway until 1957, when demethylchlortetracycline was introduced, and in the succeed- ing years yet more compounds were derived, all with similar antimicrobial activity, and showing complete cross-resistance, but differing in such properties as half life, solubility, and incidence of some unwanted effects. The most recently introduced in Britain, minocycline, does differ from other tetracyclines in its antimicrobial spectrum since it shows activity against staphylococci resistant to other tetracyclines ${ }^{1}$ and also, incidentally, against Nocardia asteroides.

The advantages offered for many years by the tetracyclines have now been eroded in a variety of ways. Antibiotic resistance has become common among pathogenic bacteria, and many Gram-negative bacilli formerly inhibited now carry $R$-factors conferring resistance both to tetracyclines and to other drugs. Resistant strains of Streptococcus pyogenes are now common enough to make tetracyclines unsuitable as first choice for infections caused by this organism, and tetracycline resistance in pneumococci is also well recognized. Even for drug-sensitive organisms tetracyclines must now compete with a variety of new wide-spectrum antibiotics with broadly comparable activities, in particular some of the semisynthetic penicillins and the cephalosporins. For staphylococcal infections yet more competitors have appeared in the form of erythromycin, the lincomycins, and fusidic acid.

Other limitations have been imposed on the use of these compounds by the emergence of several unwanted effects not recognized in the early years of the tetracycline era. Gastrointestinal side effects were described from the beginning, and the rare syndrome of liver damage from excessive dosage during pregnancy was also recognized early. ${ }^{2}$ More recently two additional serious constraints have emerged, together with several other minor or uncommon unwanted effects. The deposition of tetracyclines in developing teeth and bone leads to staining of teeth ${ }^{3}$ and temporary inhibition of bone growth. Except for very special reasons these drugs are therefore barred from use in pregnancy or in children under 7 years of age. The other important drawback is that tetracyclines by reason of their anti-anabolic action tend to precipitate or worsen renal failure. This danger, though clearly described in 1963 by Shils, ${ }^{5}$ became widely realized only some years later, ${ }^{6}$ and tetracyclines are now generally avoided in patients with known renal failure. Many patients, however, with mild and unrecognized kidney disease are at risk when tetracyclines are administered for an unrelated illness such as bronchitis. Since the worst effects are often delayed for some days the relationship between tetracycline administration and deterioration of renal function is easily missed. Doxycycline is less troublesome in this respect ${ }^{7}$ than the other compounds. Another form of renal damage, the Fanconi syndrome associated with outdated capsules, ${ }^{8}$ was related to types of excipient no longer used and is not now seen. Other less common side effects are photosensitivity, seen particularly with demethylchlortetracycline, and the rare syndrome of benign intracranial hypertension. ${ }^{9}$

These limitations have reduced the range of usefulness once enjoyed by the tetracyclines, which now occupy a slightly peculiar position in the armamentarium of antimicrobial agents. Though no longer pre-eminent for any infection common in Britain they are still very extensively used. At the same time they remain drugs of first choice for a remarkable variety of mostly uncommon or rare infections. Tetracyclines have their widest general use in acute or acute-on-chronic respiratory infections, in which they compete with ampicillin and co-trimoxazole. They are also commonly and wrongly prescribed for a variety of trivial febrile illnesses, probably mainly of viral origin and better not treated by antibiotics at all. The more specific but less common indications for a tetracycline 